\title{
THE CONTRIBUTION OF MENTAL HEALTH NURSE TO TELEPSYCHIATRIC APPLICATIONS
}

\author{
Olga Velentza ${ }^{1}$, Nasim Aouant ${ }^{2}$ \\ ${ }^{1}$ Aiginiteio Hospital, Athens, Greece \\ ${ }^{2}$ Central and Cecil Trust, London, U.K.
}

\begin{abstract}
.
Introduction: Telecommunication technology radically changes the health care delivery system. Telemedicine and telenursing have many benefits as they reduce the cost of health care without lowering the quality. They also provide high-quality medical services in deprived and remote areas, upgrading the medical services locally.

The role of the nurse concerns both the knowledge of technology and the patient's support. Also nurse undertakes the monitoring of patients with files in printed or electronic form and has knowledge of community health care, counseling, nursing and health informatics and applies them to the environment that works accordingly.

Purpose: The purpose of this study is to highlight new ways of delivering nursing care through technology aimed at the quality and effective care of health care users.

Method: An extensive review of the recent bibliography in the "Heal Link", "Google Scholar" and "Pub Med" computer databases was carried out, referring to nursing through telecommunication and electronic systems in telepsychiatry applications.

Results: Support the telemedicine and telenursing ideas continues to evolve and the evolution of technology is likely to change the way nurses will implement nursing interventions.

Many European countries have developed specific guidelines designed to provide clear guidance to nurses involved in telenursing and enhance their ability to provide safe, effective care consistent with ethical rules.

To practice the telenursing in psychiatry nurse should be equipped with additional skills and competencies related to knowledge about modern technology, the application of functional protocols and telehealth procedures, communication skills and appropriate behavior, flexibility and ability to recognize when telemedicine is not suitable for the patient's needs. It is required to provide effective nursing services, to evaluate them regularly and to fill gaps that they ought to identify.

Conclusions: Although technology is available, telemedicine and telenursing meet a plurality of obstacles to their exercise, as there is no legislation, there is no training for healthcare professionals in telematic, and there are no health care organizations in which IT is integrated, while the existing electronic systems do not guarantee quality at all stages of telematic care.

Although telenursing changes the method by which nursing services are delivered at a professional level, it does not change the nature of the nursing practice.

Telenursing is an emerging and rapidly expanding role for the profession of nurse and offers unlimited opportunities for its members.
\end{abstract}

Key words: Telenursing, Telepsychiatry, Moral ethical issues, Nursing principles 